Article

\title{
Semiperiodic Ultra-Broadband Double-Grating to Improve c-Si Thin-Film Solar Cell's Optical Absorption, through Numerical Structural Optimization
}

\author{
Mandana Jalali ${ }^{1,2, *(1)}$, Hamid Nadgaran ${ }^{2}$ and Daniel Erni ${ }^{1}{ }^{1(D)}$ \\ 1 General and Theoretical Electrical Engineering (ATE), Faculty of Engineering, University of Duisburg-Essen, \\ and CENIDE-Center for Nanointegration Duisburg-Essen, 47048 Duisburg, Germany; \\ daniel.erni@uni-due.de \\ 2 Faculty of Science, Department of Physics, Shiraz University, Shiraz 71454, Iran; nadgaran@shirazu.ac.ir \\ * Correspondence: mandana.jalali@uni-due.de; Tel.: +49-203-379-3183
}

Received: 30 April 2019; Accepted: 17 May 2019; Published: 21 May 2019

\begin{abstract}
Plasmonic gratings provide effective photon management techniques in thin-film solar cells, capable of extending the optical thickness of the solar cell's active layer. However, the ultra-broadband nature of such application makes an optimal design of the grating structure quite challenging, since a fully periodic grating operates only in specific spectral ranges. To achieve a more broadband design, semiperiodicity is introduced, which, due to having controllable disorder, is an apt solution in broadband optical applications. In this work, semiperiodic double gratings as a broadband photon management technique are introduced in order to improve the optical absorption of c-Si thin-film solar cells, and optimized through numerical structural optimization. Physical parameters of both front and back gratings are determined taking the spectrally integrated optical absorption as the figure of merit and subsequently a semiperiodic double grating is established through adding defects to the fully periodic structure. It is shown that such semiperiodic structure is capable of enhancing the spectrally integrated optical absorption $88.6 \%$ compared to a reference structure without gratings.
\end{abstract}

Keywords: grating; optical absorption; numerical structural optimization; photon management; plasmonic; semiperiodicity; solar cell

\section{Introduction}

One of the most promising solar cell designs are thin-film solar cells due to their low cost, low weight, lower recombination probability together with mechanical flexibility [1-3] and, accordingly, such structures gained a great deal of attention among researchers. The drawback of such structures is however their poor optical absorption due to the substantially reduced active material thickness [4]. In order to resolve this while improving the active layer's optical absorption, various photon management techniques have been proposed [5-7]. A well established approach aims at embedding plasmonic nanoparticles within the active layer to increase the optical absorption due to scattering and plasmonic excitation [8-10]. Another technique introduces surface roughness to the active layer resulting in extended photon path lengths due to diffuse light injection and scattering within the active layer yielding better optical absorption [11,12].

In this context, plasmonic gratings turned out to be a versatile photon management technique since they have the potential of plasmonic excitations in addition to creating strong field localizations within the active layer, which both result in a higher optical absorption probability. Moreover, such gratings diffusely backscatter the light withing the active layer and consequently extend the photon path length which is then easily translated into better optical absorption [13-15]. 
A major challenge in all mentioned photon management techniques is to efficiently exploit the ultra-large bandwidth of the solar light in the wavelength range from 350-1100 nm represented e.g., by the global standard spectrum AM1.5G. Many photon management techniques are designed based on fully periodic structures which are capable of supporting some well-defined, spectrally narrow modes and mode groups. Correspondingly, such designs are effective only in narrow spectral ranges and miss the rest of the spectrum. In contrast, techniques that are based on random structures simultaneously excite a lot of weak modes in a very broad wavelength range and, therefore, despite their large bandwidth, they will not strongly affect the absorption spectra. As proposed here, the solution should lie somewhere between full periodicity and randomness where we are going to refer to as semiperiodicity $[16,17]$.

Defining such semiperiodic structure is quite challenging and, even though there are some proposed designs for photon management, there is still room for further research to successfully define an ultra-broadband semiperiodic scattering topology [17-19]. Our approach toward defining semiperiodicity consists of adding defects to a fully periodic structure in order to introduce some degrees of randomness. Nevertheless, there is no straightforward methodology for adding such defects and hence we have used numerical structural optimization, letting the geometry to evolve itself according to given specifications, while taking the spectrally integrated optical absorption as the figure of merit.

The remainder of the paper is organized as follows. In Section 2, the details of the structure are provided together with the simulation scenario and afterwards, in Section 3, a fully periodic plasmonic back grating is designed and optimized. In Section 4, a fully periodic front grating is added to the structure and its effect on the overall absorption performance is thoroughly investigated, while, in Section 5, our semiperiodicity idea is elucidated and various techniques considering a proper definition of semiperiodicity are discussed. The optimized semiperiodic double grating (DG) structure is then compared in Section 6 with semiperiodic structures based on deterministic mathematical semiperiodic sequences, and finally, in Section 7, conclusions are drawn. The proper optimization algorithm namely the utilized breeder evolutionary algorithm is presented in Appendix A.

\section{Solar Cell Design and Simulation}

The design of the whole underlying c-Si thin-film solar cell structure is displayed in Figure 1, and consists of a $400 \mathrm{~nm}$ thick crystalline silicon absorbing layer, without doped layers, covered with a $100 \mathrm{~nm} \mathrm{Si}_{3} \mathrm{~N}_{4}$ anti-reflection coating on top. The reason for such choice of anti-reflection coating is the appropriate material refractive index for the optimal anti-reflective effect that emerges from a layer with a $100 \mathrm{~nm}$ thickness that will completely cover the front grating. The back electrode is a $100 \mathrm{~nm} \mathrm{Ag}$ layer, where Ag is chosen since the back grating material is also Ag and hence the fabrication process would be easier. It should be mentioned that the top electrode is not included in the simulations, since it has no optical effect on the considered layer stack respectively on the scattering mechanisms in the resulting solar cell topology.

A periodic plasmonic binary Ag back grating with $45 \mathrm{~nm}$ tooth height, $175 \mathrm{~nm}$ tooth width and a $525 \mathrm{~nm}$ periodicity is implemented at the back of the active layer, respective on top of the back electrode. The parameters have been optimized using an evolutionary search heuristics and slightly modified for feasibility reasons. The front grating consists of $\mathrm{c}-\mathrm{Si}$, similar to the active layer material, and its physical parameters are chosen based on a direct correlation with the periodic back grating. Our semiperiodic gratings have the same physical parameters as the periodic ones, while just omitting/altering teeth when adding defects.

Furthermore, the excitation is given by a perpendicularly impinging broadband plane wave (covering the spectral range of 350-1100 nm) containing both s- and p-polarization mimicking an AM1.5G solar illumination. The numerical analysis of the overall solar cell is carried out using the simulation platform COMSOL Multiphysics (together with the Wave Optics Module), which is a Finite-Element-Method (FEM) based software. For the optimization procedure, the COMSOL 
Multiphysics LiveLink ${ }^{T M}$ for MATLAB has been applied since the optimization code is written in a MATLAB script. This results in slightly longer simulation times compared to the COMSOL Multiphysics optimization package; however, we favor this approach due to the better control over the optimization procedure.

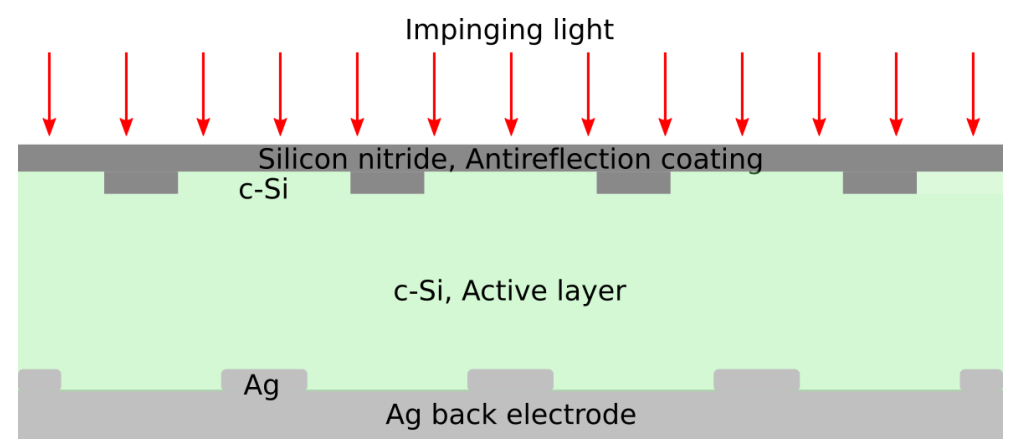

Figure 1. schematic of the solar cell structure.

\section{Periodic Plasmonic Back Gratings}

A common photon management technique relies in adding a periodic plasmonic back grating to the active layer since such grating improves the optical absorption through various scattering channels while simultaneously acting as the back electrode. This back grating may excite several plasmonic modes yielding strong field localizations within the active layer, which improves the optical absorption in the corresponding spectral range. Furthermore, the presence of such artificial roughness in the back of the active layer results in diffuse scattering of the escaped light back to the layer and hence extending the photon path length.

Considering all the mentioned arguments, an Ag rectangular (i.e., binary) back grating is implemented at the back of the structure. The physical parameters of the grating, namely the grating period, tooth height and tooth width determine its functionality regarding to the number and spectral positions of the modes which are excited. Considering the intended ultra-broadband performance of the solar cell, where a spectral range between $350-1100 \mathrm{~nm}$ of an AM1.5G illumination spectrum is addressed, the mentioned parameters cannot be designed for only a narrow spectral range. In this regard, the grating parameters (such as grating tooth height, width and periodicity) were subjected to optimization where the spectrally integrated optical absorption acted as the quality respective the fitness function. The optimization was terminated after about 800 iterations when the final population reached a certain level of quality and afterwards the best performing structure was analyzed. The optimized structure absorbs $30.2 \%$ of the total illuminated power, which is $50.2 \%$ higher compared to the same structure without periodic back grating.

The simulated spectral response of the active layer's optical absorption in the structure with optimized periodic Ag back grating compared to a similar structure without any back grating is depicted in Figure 2a. It can be seen that various new peaks emerged in the absorption spectra due to the presence of the back grating. To study the reason behind each peak, the electric field distribution at the corresponding wavelengths are investigated and illustrated in Figure 2b, where the first peak is associated to a plasmonic mode that is excited around each tooth. The second and third peaks refer to similar strong field localizations, which also resulted in improved optical absorption. However, it should be mentioned that all the peaks appear as quite narrowband. 


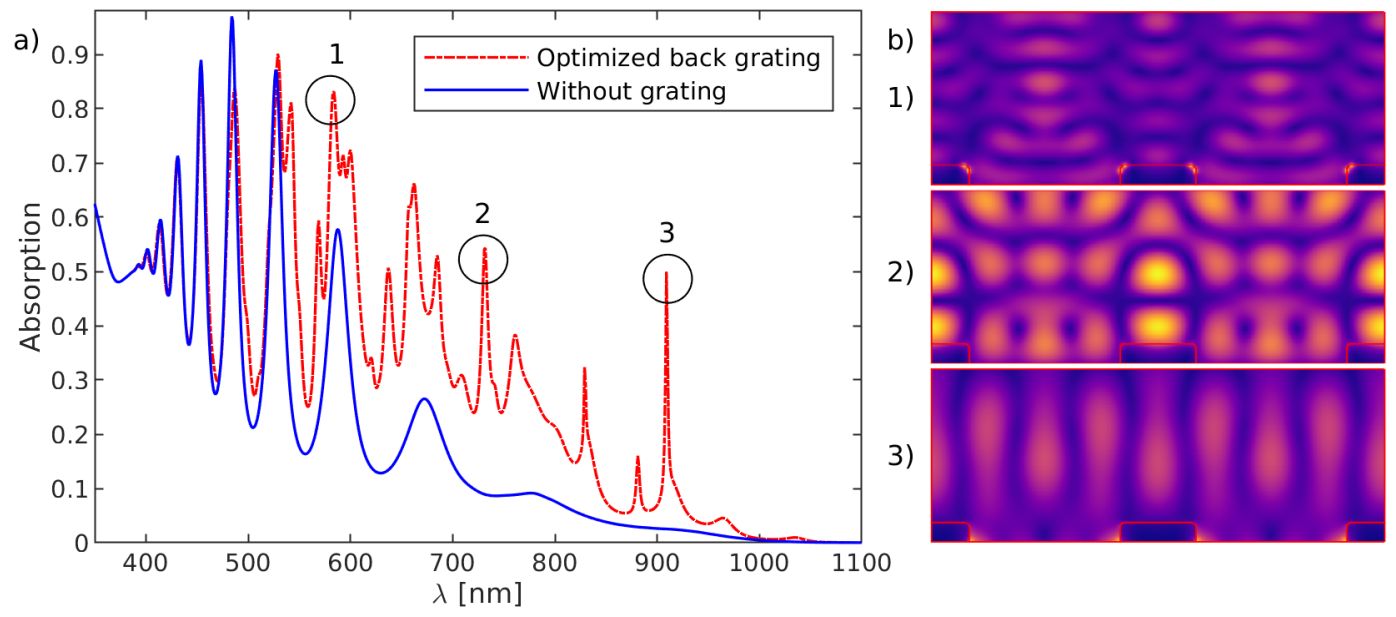

Figure 2. (a) simulated spectral response of the active layer's optical absorption in structures without periodic back grating (solid blue line) and with optimized periodic back grating (red dash-dotted line); (b) simulated electric field distributions at the operating wavelengths as labelled in the graph (a).

\section{Periodic Double Gratings}

Adding a front grating will further improve the solar cell performance owing to various mechanisms. The front grating can be regarded as a controllable surface roughness, which, through deviating the incident light direction, extends the photon path within the active layer. Surface roughness by diffusely transmitting the incoming light into the active layer at angles smaller than those of total internal reflection trap the light inside the active layer, when such back and forth scattering at interfaces further extends the photon path four or five times within the active layer [20].

Despite having a $100 \mathrm{~nm} \mathrm{Si}{ }_{3} N_{4}$ anti-reflection coating on top of the active layer, a considerable amount of the illuminated power is still reflected and hence lost without entering the active layer. Another aspect of adding a front grating to the active layer is to further reduce such reflection as the front grating creates an intermediate layer with refractive index amid the anti-reflection coating and the active layer [21]. This results in the formation of a graded refractive index layer stack which consequently improves the incoupling performance [22-26].

Additionally, each tooth acts as an elementary light source that injects diffused light into the active layer while various modes and mode groups are excited within the active layer. Constructive interferences among the excited modes in the front and back gratings would result in strong field localizations (i.e., hot spots) which may directly correspond to strong peaks in the optical absorption spectra. However, destructive interferences are also possible and hence a careful design of front grating parameters along with its phase relative to the back grating is needed to minimize the effect of such destructive interferences.

The first important parameter in designing a front grating is the material. Plasmonic materials cannot be positioned on top of the active layer due to their high absorption and correspondingly high loss. Since creating a new interface between the active layer and the front grating would result in higher reflection, in this work, the front grating material is chosen to be c-Si, which corresponds to the active layer material. Such design would also result in easier fabrication. As the main criteria for the front grating parameters are their direct correlation with the back grating, the front grating's physical parameters, namely tooth height and width are considered to be related to the corresponding optimized values of the back grating and hence are chosen accordingly. The front grating parameters are either the same, double or half of the back grating parameters. The front grating phase relative to the back grating (i.e., the grating offset) is a critical parameter that determines the front-back gratings coupling. Two cases of in-phase and out-of-phase front gratings are considered where in-phase means that each front grating tooth is positioned at the exact same position of the back grating tooth, while out-of-phase refers to a front grating tooth that is positioned in between two back grating teeth. 
The percentage of spectrally integrated absorbed power along with the reflected power relative to the total illuminated power for the mentioned double grating structures are presented in Table 1 , which shows that the best performing structure $(45 \mathrm{~nm}$ tooth height, $350 \mathrm{~nm}$ tooth width and in-phase with the back grating) improves the spectrally integrated optical absorption by $20.9 \%$ compared to the case with only a back grating. The spectral response of the active layer's optical absorption for the mentioned structure together with the structure with only a back grating and without any grating are depicted in Figure 3a. One interesting feature of this graph is the prominent enhancement of the optical absorption in the spectral range $350-450 \mathrm{~nm}$ which is mainly due to the reduced reflection. In the Figure $3 b-1$, the electric field distribution at a typical wavelength in this range is illustrated which clearly shows strong light injection into the active layer through the front grating. Studying the electric field distributions at other peaks in the optical absorption validates the fact that strong field localizations happen as a consequence of front grating presence (cf. Figure $3 b-2,3$ ).
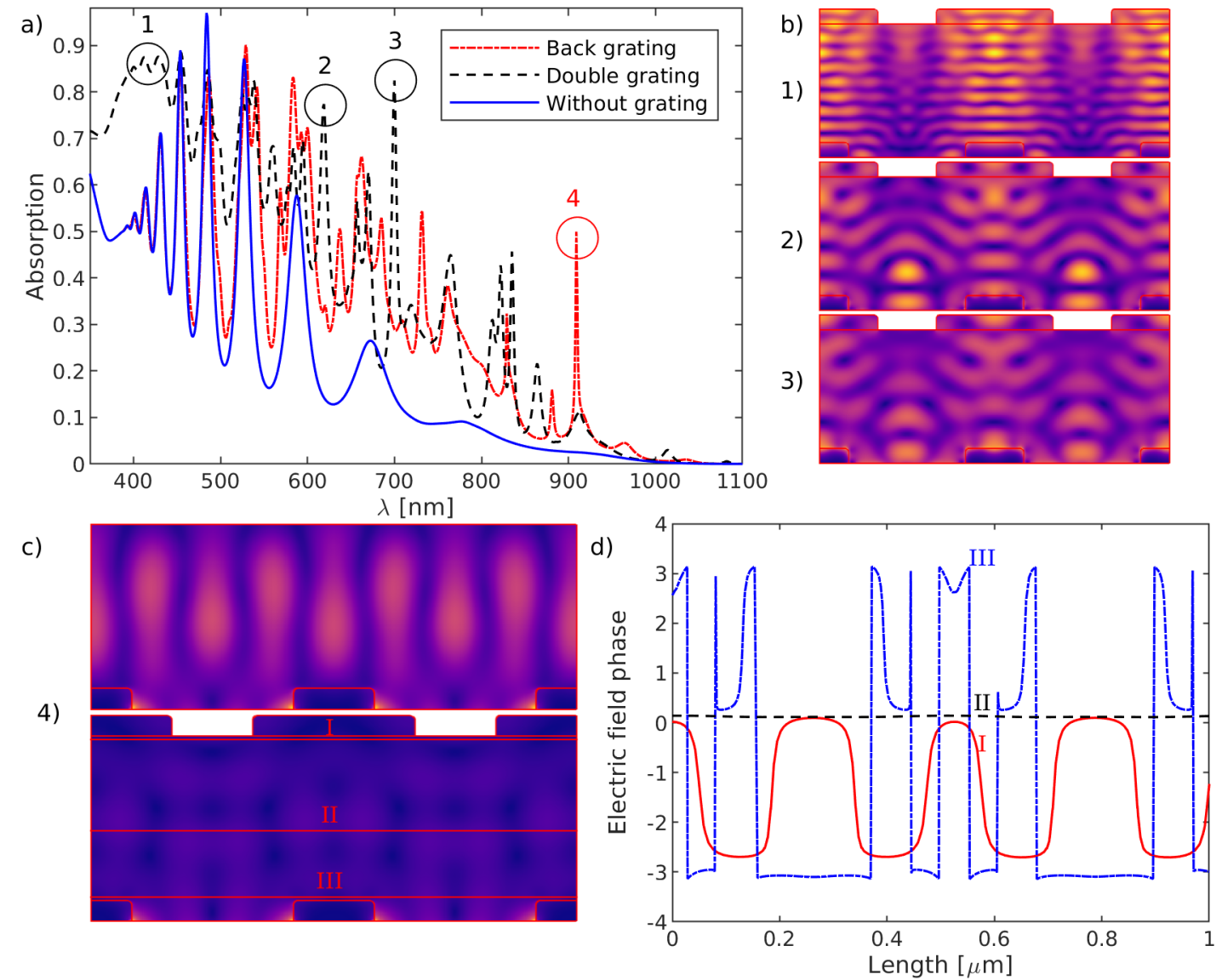

Figure 3. (a) simulated spectral response of the active layer's optical absorption in structures without any grating (solid blue line), with an optimized periodic back grating (red dash-dotted line) and with a periodic double grating (black dashed line); (b) simulated electric field distributions at three first labelled operating wavelengths in the graph (a); (c) simulated electric field distributions at the fourth labelled operating wavelength in the graph (a), for structures with a periodic back grating and a periodic double grating; (d) phase of the electric field at the fourth labelled operating wavelength in the graph (a), for the structure with a periodic double grating displayed at the front grating (I), in the middle of the active layer (II) and at the back grating (III). 
Table 1. The percentage of spectrally integrated absorbed power as well as the reflected power relative to the total illuminated power for the periodic double grating designs together with structures without a grating and with only a periodic back grating.

\begin{tabular}{|c|c|c|c|c|}
\hline & Width (nm) & Height (nm) & Absorbed Power & Reflected Power \\
\hline Without grating & - & - & $20.1 \%$ & $78.7 \%$ \\
\hline Back grating & 175 & 45 & $30.2 \%$ & $42.9 \%$ \\
\hline \multirow{6}{*}{ Double grating, in-phase } & \multirow{2}{*}{350} & 45 & $36.5 \%$ & $35.0 \%$ \\
\hline & & 22 & $33.6 \%$ & $39.8 \%$ \\
\hline & \multirow{2}{*}{175} & 45 & $33.0 \%$ & $38.7 \%$ \\
\hline & & 22 & $31.4 \%$ & $43.9 \%$ \\
\hline & \multirow{2}{*}{87} & 45 & $32.3 \%$ & $40.5 \%$ \\
\hline & & 22 & $31.0 \%$ & $44.8 \%$ \\
\hline \multirow{6}{*}{ Double grating, out-of-phase } & \multirow{2}{*}{350} & 45 & $34.5 \%$ & $35.7 \%$ \\
\hline & & 22 & $32.0 \%$ & $37.9 \%$ \\
\hline & \multirow{2}{*}{175} & 45 & $32.5 \%$ & $42.9 \%$ \\
\hline & & 22 & $30.6 \%$ & $42.9 \%$ \\
\hline & \multirow{2}{*}{87} & 45 & $31.9 \%$ & $45.3 \%$ \\
\hline & & 22 & $30.6 \%$ & $43.8 \%$ \\
\hline
\end{tabular}

It should also be mentioned that in some cases the absorption is reduced which is mainly due to destructive interference between the front and back gating. An example of such destructive interference emerges at $910 \mathrm{~nm}$ as indicated in Figure 3a. The electric field distribution at the corresponding wavelength for the structures with a periodic double grating and only a periodic back grating is shown in Figure $3 c$ together with the phase of the electric field within the active layer (cf. Figure 3d). The destructive interference between the back and front gratings resulted in a weak electric field distribution throughout the active layer and consequently in poor absorption.

As discussed and already shown in Table 1, the presence of a front grating substantially reduces reflection. This reduction is even more pronounced at oblique illuminations, which is advantageous as reflection is a major issue in the solar cell's performance at these oblique incidences. Another advantage of the front grating as the anti-reflection coating is its inherent tendency toward omnidirectionality. In Figure 4, the spectral response of the reflected power for an illumination angle of 0 and $\frac{\pi}{8}$ are plotted in comparison to the structure with only a back grating, where it can be seen that the presence of the front grating reduced the reflection at both illumination angles in particular in the short wavelength regime where c-Si is a good absorber.

The front grating's tooth height is a deterministic parameter which affects the performance of the front grating as both, a light coupler and an anti-reflection coating. In order to reduce backscattering, the grating tooth should not be too high and hence values around $90 \mathrm{~nm}, 45 \mathrm{~nm}$ and $22 \mathrm{~nm}$ are investigated aiming at an improved light coupling to the active layer. As expected, the $90 \mathrm{~nm}$ tooth height doesn't show an improvement in the optical absorption since it backscatters a huge fraction of light and shows a $26.1 \%$ higher reflection compared to the structure with only a back grating. In addition, a $22 \mathrm{~nm}$ tooth height is not capable of exciting various modes and conclusively a $45 \mathrm{~nm}$ tooth height which is identical to the back grating's teeth shows both, better anti-reflection capabilities and strong mode excitations within the grating (cf. Figure 5). 



Figure 4. simulated spectral response of the reflected power in structures with a periodic back grating (red dash-dotted line) and with a periodic double grating (blue line) at (a) normal incidence and at (b) $\frac{\pi}{8}$ tilted incidence.
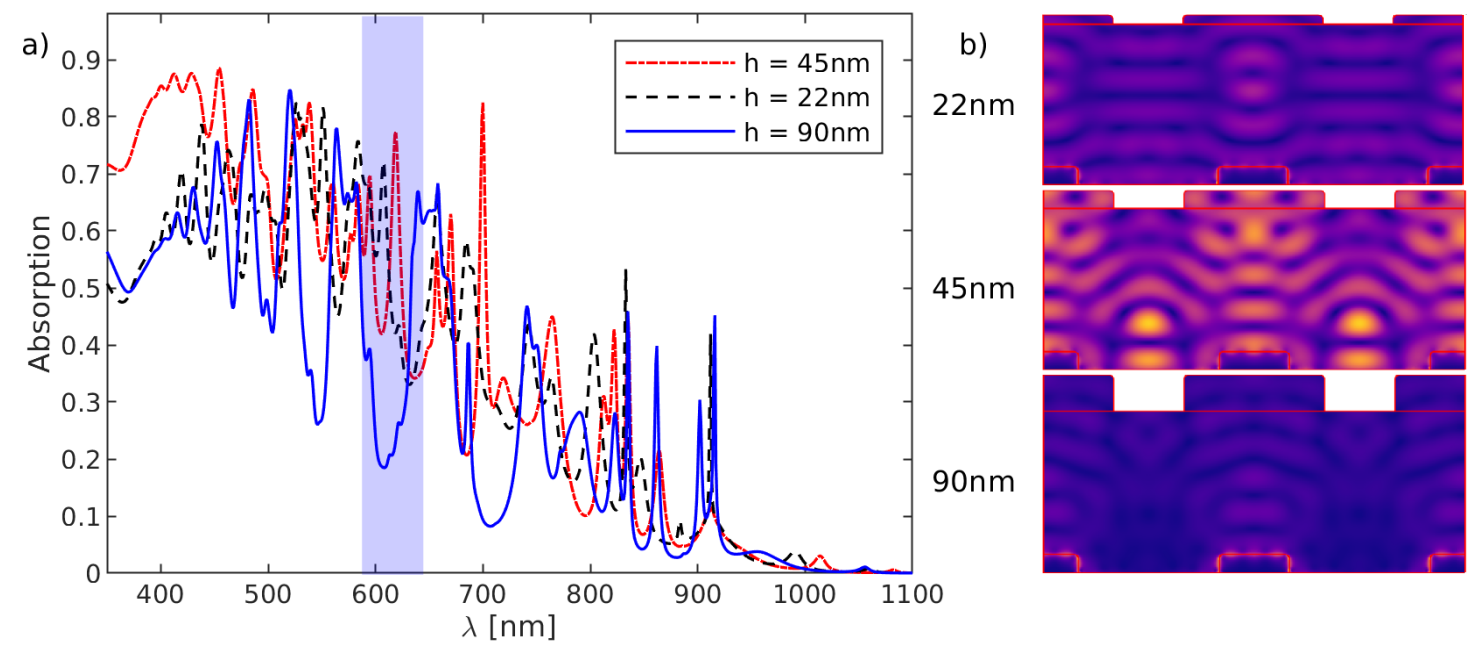

Figure 5. (a) simulated spectral response of the active layer's optical absorption in structures with a periodic double grating and tooth heights of $90 \mathrm{~nm}$ (solid blue line), $45 \mathrm{~nm}$ (red dash-dotted line) and $22 \mathrm{~nm}$ (black dashed line); (b) simulated electric field distributions at the operating wavelength of $620 \mathrm{~nm}$ highlighted in the graph (a), for all mentioned tooth heights.

The front grating's tooth width should also be chosen with care in order to achieve a strong light coupling to the active layer. Correspondingly, values such as $350 \mathrm{~nm}, 175 \mathrm{~nm}$ and $87 \mathrm{~nm}$ for the tooth widths are simulated and the spectral response of the active layer's optical absorption along with the reflected power are illustrated in Figure 6. We can now deduce that a $350 \mathrm{~nm}$ width has the lowest reflection and hence results in better optical absorption. In order to validate that the improvement in the optical absorption is not simply the result of a slightly increased active material volume due to the presence of the front grating, the volume density of the optical absorption is calculated for all structures showing the same improvement as the optical absorption. 

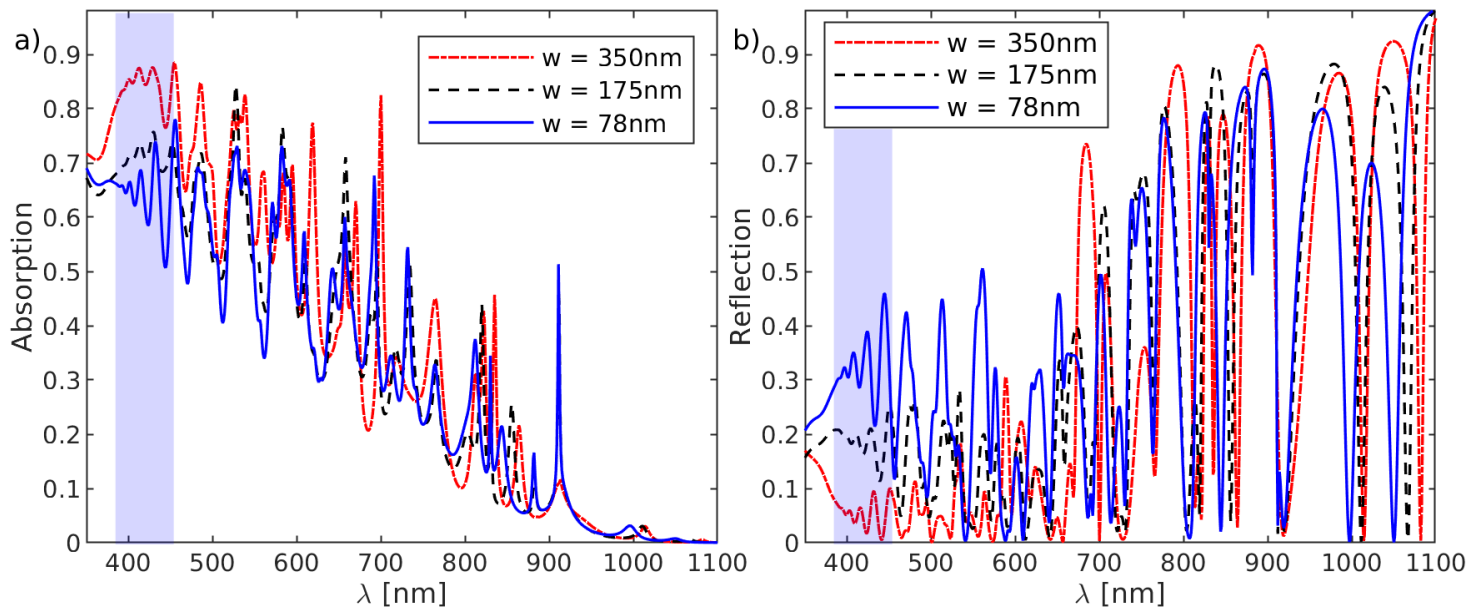

Figure 6. (a) simulated spectral response of the active layer's optical absorption in structures with a periodic double grating and tooth widths of $78 \mathrm{~nm}$ (solid blue line), $175 \mathrm{~nm}$ (black dashed line) and $22 \mathrm{~nm}$ (red dash-dotted line); (b) simulated spectral response of the reflected power for all the mentioned tooth widths.

For an optimal periodic double grating design, the effective coupling between front and back gratings is vital. The relative phase of the front grating in regard to the back grating is the determining parameter that should be carefully chosen according to a predominate number of constructive interferences in the desired spectral range compared to the number of destructive interferences. Two cases of in-phase and out-of-phase front gratings concerning possible front-back grating coupling are studied and the results are depicted in the Figure 7. The spectrally integrated optical absorption in the case of the periodic in-phase double grating is about 5.8\% higher than for the out-of-phase case, which is due to a better front-back gratings coupling.


Figure 7. (a) simulated spectral response of the active layer's optical absorption in structures with only a periodic back grating (solid blue line), an out-of-phase double grating (black dashed line) and an in-phase double grating (red dash-dotted line); (b) simulated spectral response of the reflected power for all mentioned structures. 


\section{Semiperiodicity}

Semiperiodicity or aperiodicity is a concept basically meaning order without periodicity, and became a hot topic in various fields such as e.g., mathematics, physics, biology and economy [27]. Traditionally, systems are divided into two groups of periodic and non-periodic structures and are treated accordingly. However, there is a rich field in between that is associated with semiperiodicity and can be categorized based on their degree of order respective to their underlying spatial correlations. Semiperiodic structures have various potential applications especially in broadband optical systems $[17,28]$, since they lie somewhere between full periodicity and randomness and therefore can provide a trade-off between the expected performances of these two characteristic topologies. Fully periodic structures consist of repeated building blocks respective unit cells in space and hence display long range order which corresponds to the excitation of strong but narrowband modes. In comparison, random structures are capable of simultaneously exciting several modes and mode groups, which are quite broadband however weak. Semiperiodic structures have some degrees of order and although each realization will not behave exactly as the other, there would be some correlations amongst these realizations and consequently such structures can sustain modes that are both not as narrow as in fully periodic structures and not as weak as in random structures, and hence provide a nice compromise for broadband optical applications.

Designing semiperiodic structures is nonetheless quite challenging since there is no explicit way of capturing semiperiodicity in a structure and accordingly there is some research regarding the proper definition of semiperiodicity $[19,29,30]$. The approach that is used here is adding defects to a fully periodic grating in order to "move" toward randomness. Adding defects means omitting teeth in the fully periodic grating. The idea is schematically illustrated in Figure 8, where the first structure in Figure $8 \mathrm{~b}$ is fully periodic; in the second structure, some defects are added based on an already existing mathematical semiperiodic sequence, namely the Thue-Morse sequence [31]. The Thue-Morse sequence is mapped in parallel onto both periodic gratings with commonsurable lengths, where the zeros of the Thue-Morse sequence are labeling the locations of the missing teeth. The defects are added to both, front and back grating, where the back grating is mapped based on the Thue-Morse sequence while the front grating is processed according to the bitwise negation of the Thue-Morse sequence (i.e., each bit has the opposite value of the same bit in the binary representation of the sequence) [17]. The third structure is our fully optimized semiperiodic double-grating structure resulted from numerical structural optimization where both front and back gratings were subjected to optimization.

a)



b)

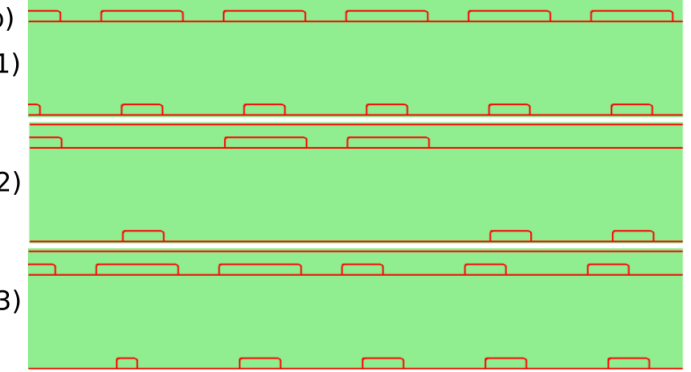

Figure 8. (a) schematic illustration of semiperiodicity; (b) geometry of a periodic (1) , a Thue-Morse semiperiodic (2) and an optimized semiperiodic double grating (3).

\section{Semiperiodic Double Gratings}

A well-designed semiperiodicity would allow the capturing of the solar illumination in its broad spectral range. This idea is first tested through defining semiperiodicity based on corresponding mathematical sequences, such as Thue-Morse, Fibonacci and Rudin-Shapiro sequences [17,31]. The results in terms of the spectral response of the active layer's optical absorptions are depicted in Figure $9 \mathrm{a}$ for a wavelength range of $700-800 \mathrm{~nm}$. Here, a narrow but strong peak in the periodic 
double grating is transformed into a broad though weaker absorption which applies for all semiperiodic sequences. Through analyzing the electric field distribution at the corresponding operating wavelength (cf. Figure 9b), it can be conferred that, in all semiperiodic cases, the electric field is dispersed through the whole active layer instead of being localized in some hot spots, indicating that the entire active material volume is contributing uniformly to the optical absorption.

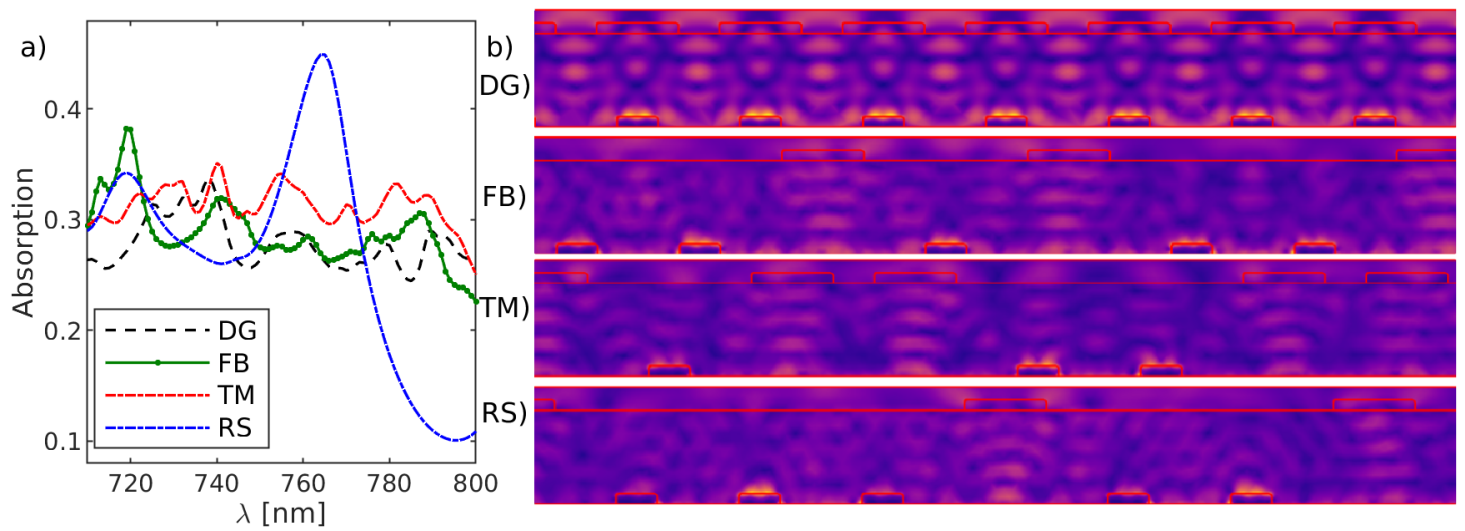

Figure 9. (a) simulated spectral response of the active layer's optical absorption in structures with a periodic double grating (DG) along with double gratings based on Fibonacci (FB), Thue-Morse (TM) and Rudin-Shapiro (RS) sequences; (b) simulated electric field distributions at an operating wavelength of $764 \mathrm{~nm}$ for all of the mentioned structures.

Defining semiperiodicity based on mathematical sequences, despite showing the advantages of such approach, is not enough to reach an optimal design for an ultra-broadband solar cell. To reach such design, a breeder evolutionary algorithm is used to create fully semiperiodic double grating purely based on this global optimization scheme. The details of the optimization procedure are provided in Appendix A. In this approach, the position of each defect as well as each tooth width (tooth widths have either half or the same value as in the periodic design) are determined by the optimization, considering a maximal spectrally integrated optical absorption as the optimization criterion. In addition, no direct correlation between back and front grating is taken into account so the geometry would totally evolve by itself. The optimization procedure was terminated after about 1020 iterations when the best performing structures within the final population achieved a good approximation to the given specifications. The fitness functions of best, worst and average individuals in the population for each iteration step are illustrated in Figure 10. This evolution clearly shows the wide range of possibilities that were explored by this global optimization procedure. The fitness of each structure is given by the percentage of spectrally integrated optical absorption relative to the total illuminated power.

The best performing of the optimized semiperiodic double gratings achieved a promising spectrally integrated optical absorption that is $88.6 \%$ higher compared to the same structure without any grating. The quantitative values for the percentage of spectrally integrated absorbed power relative to the total illuminated power for all semiperiodic double grating designs are provided in Table 2, which states that the optimized semiperiodic double grating provides the highest spectrally integrated optical absorption. To comprehend the underlying mechanisms that contribute to the performance of the optimized structure, the simulated spectral response of the active layer's optical absorption is displayed in Figure 11 in comparison to the corresponding spectra of the semiperiodic structures based on the mathematical sequences as well as for the fully periodic double grating. The comparison between the spectral responses of the active layer's optical absorption shows that, although the optimized structure doesn't show strong peaks, it maintains a nice compromise between peak strength and peak bandwidth, which is advantageous in such broadband application. 


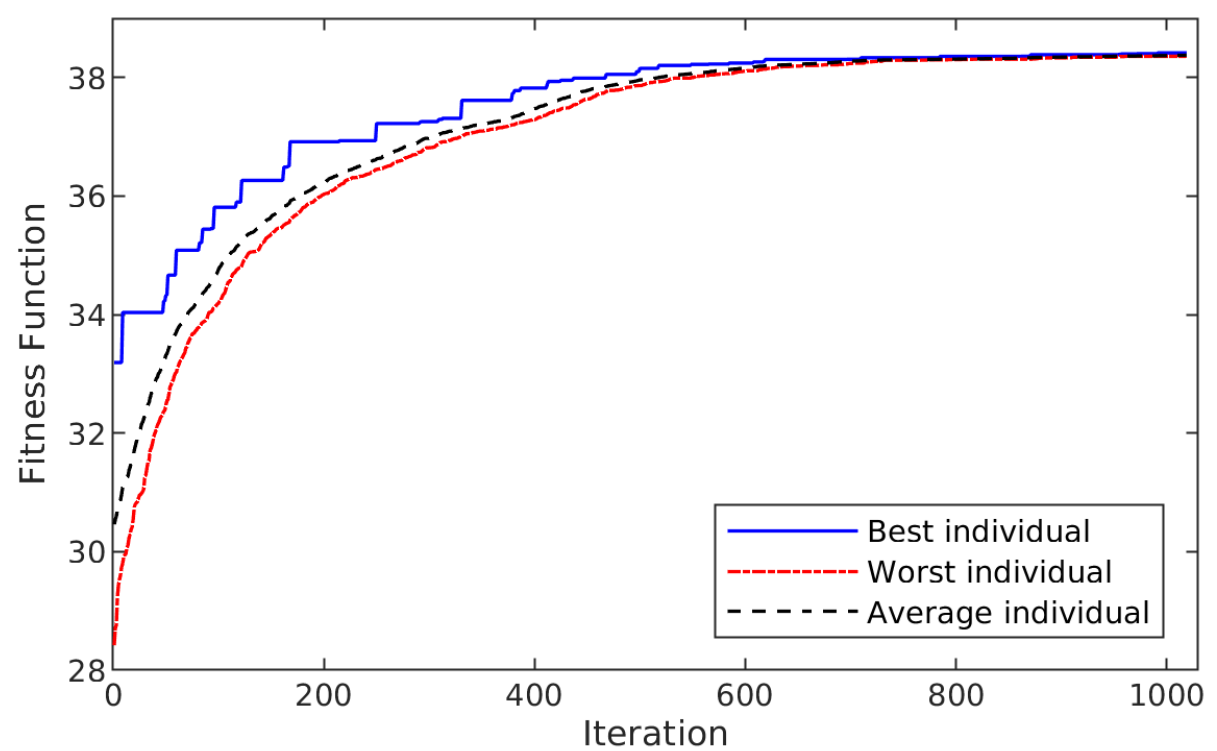

Figure 10. evaluation of optimized structures: the curve depicts the best, worst and average individual at each iteration step.

Table 2. The percentage of spectrally integrated absorbed power relative to the total illuminated power for the semiperiodic double grating designs.

\begin{tabular}{ccccc}
\hline & Fibonacci Grating & Thue-Morse Grating & Rudin-Shapiro Grating & Optimized Grating \\
\hline Absorbed Power & $31.8 \%$ & $32.6 \%$ & $32.4 \%$ & $37.9 \%$ \\
\hline
\end{tabular}


Figure 11. (a) simulated spectral response of the active layer's optical absorption in structures with double gratings, such as the optimized semiperiodic double grating, the double grating based on Fibonacci (FB), Thue-Morse (TM) and Rudin-Shapiro (RS) sequences; (b) comparison between the optimized semiperiodic double grating and the fully periodic double grating.

There are various interesting features when comparing the optimized semiperiodic double grating structure's optical absorption with the performance of the fully periodic one, which are highlighted in Figure $11 \mathrm{~b}$, together with the corresponding electric field distributions that are analyzed and depicted in Figure 12. The first characteristic feature emerges around $583 \mathrm{~nm}$ where the semiperiodicity resulted in an even stronger absorption peak compared to the fully periodic one. The electric field distributions of both double grating structures show that the semiperiodicity in the front grating resulted in better and more diffused light coupling to the active layer and consequently the optical 
absorption is increased (cf. Figure 12a). The same analysis at $764 \mathrm{~nm}$ depicts that in this case the electric field is more dispersed through the active layer which corresponded to the transformation of a strong peak in the periodic case into a broad absorption band (cf. Figure 12b). At $1014 \mathrm{~nm}$, the effective break of periodicity in the underlying structure dispersed the light even better throughout the active layer where in addition a stronger coupling among the back grating teeth has occurred yielding stronger optical absorption (cf. Figure 12c).

a)
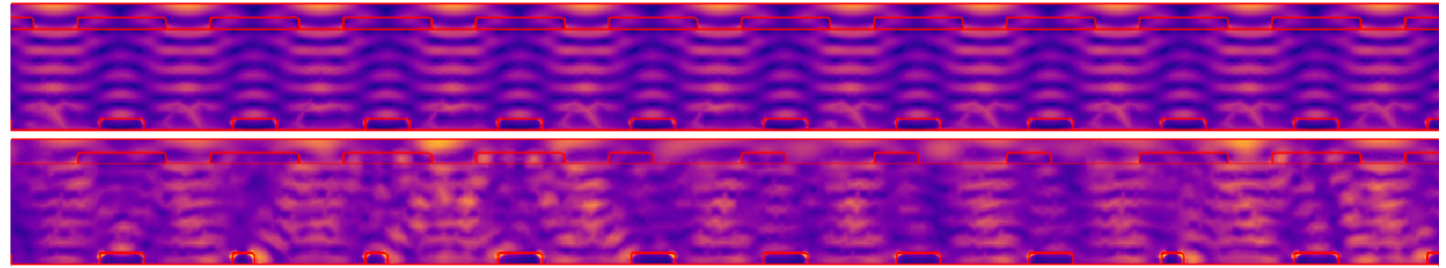

b)
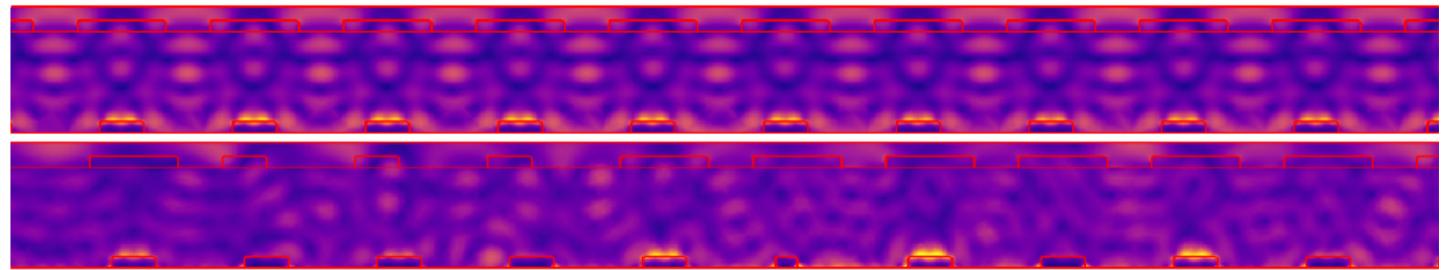

c)



Figure 12. simulated electric field distributions at different operating wavelengths of (a) $583 \mathrm{~nm}$; (b) $764 \mathrm{~nm}$; and (c) $1014 \mathrm{~nm}$ highlighted in Figure 11, for solar cell topologies with a periodic double grating (top) and an optimized semiperiodic double grating (bottom).

To be able to reliably deduce that the optimized semiperiodic double grating structure achieved a dispersed optical absorption throughout the whole active layer, a comprehensive display of the active layer's overall optical absorption has been generated in the form of a two-dimensional (2D) spatio-spectral (normalized) absorption density map that is depicted in Figure 13a. This map is complemented by two insets showing the spatial integration of the map (i.e., the frequency response of the overall optical absorption) in Figure $13 \mathrm{~b}$ as well as the spectral integration of the map (i.e., the spatial distribution of the overall absorption) in Figure 13c. Please note that the full 2D integration of the absorption density map refers to the fitness value that has been maximized within optimization. Given this sort of holistic display of the optimized solar cell's performance allows now to verify that the achieved semiperiodicity renders the resulting absorption to be distributed in the best possible way i.e., showing the intended broadband features in the spectral response together with a virtually uniform exploitation of the active layer volume. 


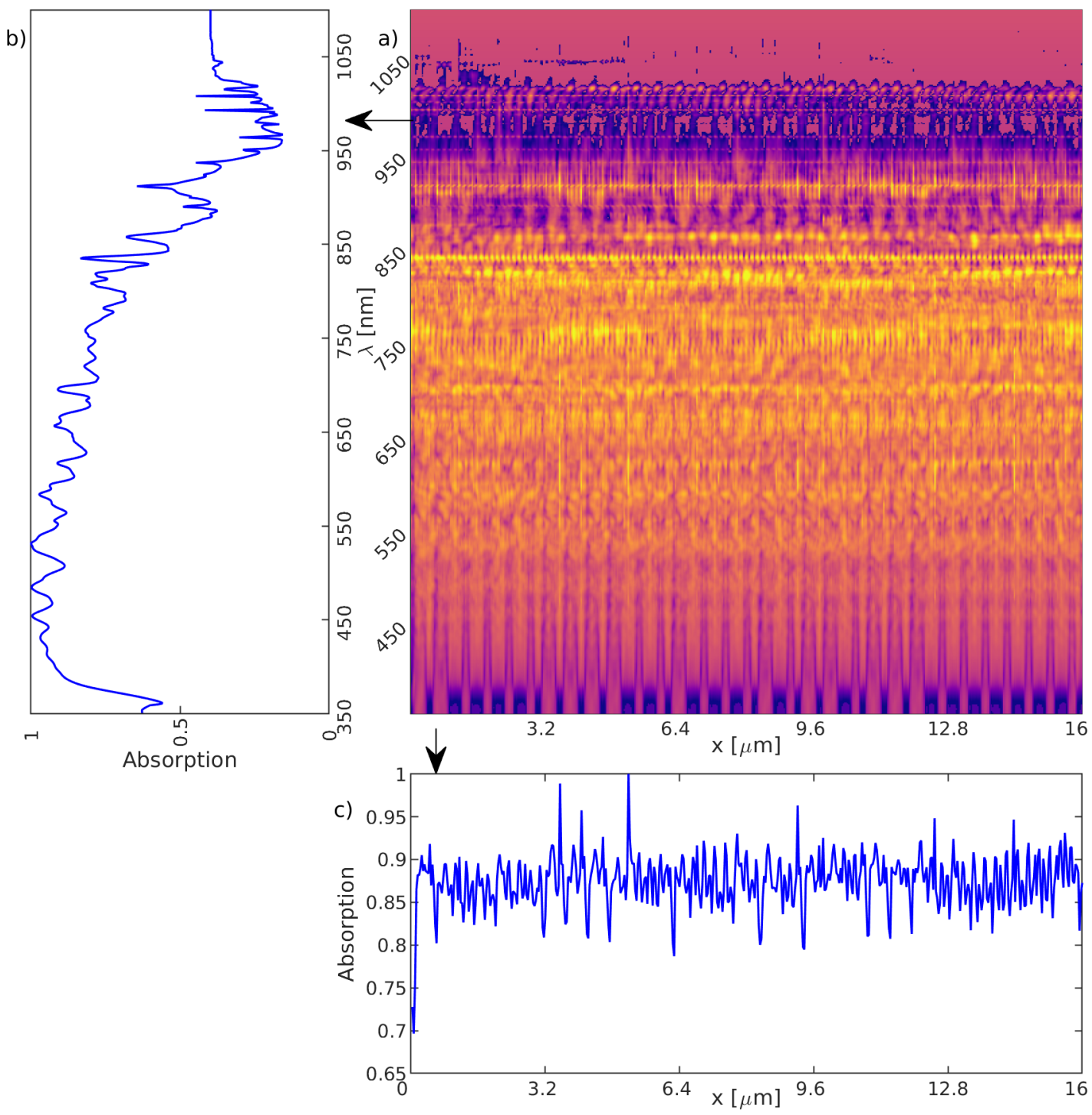

Figure 13. (a) the spatio-spectral absorption density map as a comprehensive display of the active layer's optical absorption as a function of both, the horizontal active layer position and the operating wavelength. It should be mentioned that bright colors refer to high values and cold colors to lower values; (b) spectral response of the overall absorption (i.e., spatial integration of the map); (c) spatial distribution of the overall absorption along the grating structure (i.e., spectral integration of the map).

\section{Conclusions}

In this work, the concept of semiperiodicity as an apt solution to broadband optical applications is introduced and used to improve spectral response of the optical absorption in c-Si thin-film solar cells. It is shown that using a well-defined semiperiodic grating structure can result in an effective exploitation of the active material in the spatial as well as in the spectral domain.

An optimized periodic plasmonic back grating as a first example of a photon management technique is implemented in the active layer taking the spectrally integrated optical absorption as the figure of merit. Results show that the designed grating improves the solar cell's performance up to $50.2 \%$, compared to a bare solar cell due to the excitation of several well-defined modes within the active layer.

As the next step, a periodic c-Si front grating is added to the discussed topology, which acts as an omnidirectional anti-reflection coating as well as a light coupler to the active layer and hence the active layer's optical absorption is further improved. It is shown that physical parameters of the underlying 
front grating are key in order to establish a constructive coupling between the two gratings. However, the resulted spectral response of active layer's optical absorption shows mainly strong but narrowband peaks, which leaves considerable part of the solar spectrum unused.

Applying now the concept of semiperiodicity to the design of semiperiodic double gratings, a more efficient spectral response of the active layer optical absorption is achieved, since the semiperiodic double grating is capable of creating broad optical absorption bands. The main reason lies in the broad dispersion of the electric field throughout the whole active layer instead of having some localized hot spots. Our approach towards defining semiperiodicity is adding defects in the sense of missing teeth into the periodic double grating, which, at the first stage, were introduced through mathematical semiperiodic sequences. To accomplish an optimal design, the semiperiodic sequence is realized through a numerical structural optimization ascertaining the best optical performance for the underlying solar cell structure. The proposed structure enhanced the spectrally integrated optical absorption up to $88.6 \%$ compared to a bare reference structure without gratings. Our design approach underpins the fact, that the performance of the best semiperiodic double grating topology is strongly related to a virtually uniform spatio-spectral exploitation of the solar cell's active material stack. This is not very surprising; however, it aims at a more holistic view to broadband photonic design, namely at spatio-spectral performance measures with complex interdepenencies that are only manageable in the framework of numerical structural optimization.

Author Contributions: Conceptualization, M.J., D.E. and H.N.; Methodology, M.J. and D.E.; Software, M.J.; Validation, M.J., D.E. and H.N.; Formal Analysis, M.J.; Investigation, M.J.; Resources, M.J. and D.E.; Data Curation, M.J. and D.E.; Writing—Original Draft Preparation, M.J.; Writing—Review and Editing, M.J., D.E. and H.N.; Visualization, M.J.; Supervision, D.E. and H.N.; Project Administration, D.E.

Funding: This research received no external funding.

Conflicts of Interest: The authors declare no conflict of interest.

\section{Appendix A. Breeder Evolutionary Algorithm}

Evolutionary algorithms (EA) belong to a subset of evolutionary computation, representing a population-based metaheuristic optimization scheme. The EA uses mechanisms inspired by biological evolution, namely reproduction, operators such as selection, cross-over, and mutation. Candidate solutions to the optimization problem are represented by their genotypes (i.e., parameter strings) and play the role of individuals in a population, which is evaluated by a the fitness function that determines the quality of each solution. Evolution of the population then takes place after the repeated application of the above operators as sketched in Figure A1 for a breeder EA. Breeder EAs have been used in science and engineering as adaptive algorithms for solving practical inverse problems and as simplified computational models of natural evolution [29,32-34]. There are many different versions of EAs, though the common underlying idea behind all of these techniques is the same: given a population of individuals, the environmental pressure (i.e., the design specifications) causes natural selection to become effective yielding a rise in the fitness of the population, while the latter enables a set of solutions that becomes increasingly competent with respect to the given specifications. Each individual represents a search point in the space of potential solutions to the given problem.

Having a quality function (i.e., the fitness) at hand, which determines the performance of each individuals, a set of possible solutions is randomly created and thus subsequently evaluated, yielding the individual's proper fitness. The higher the fitness, the better is the performance of the individual solution. Based on such evaluation, some of the good solutions are more probable to seed the next generation through recombination. Recombination is an operator that first includes the selection (i.e., roulette wheel selection) of two (or more) individuals which are called parents. These will give rise to two children by using (two-point) cross-over and subsequent mutation applied to the children's genotype. These offsprings will then be included into the existing population if their fitness is within the ranks of population fitness. The process is iterated until an individual with sufficient quality is found or other termination criteria are met. There are two fundamental forces that are essential to 
evolutionary algorithms. First mutation provides the required diversity and thereby facilitates novelty, and second the selection and cross-over act as the force pushing quality by balancing inheritance and combination of potentially successful characteristics. Such recombination continuously improves the fitness in the population during optimization. An overview of the proper implementation of such breeder EA is e.g., given in $[29,34]$ and the references therein.

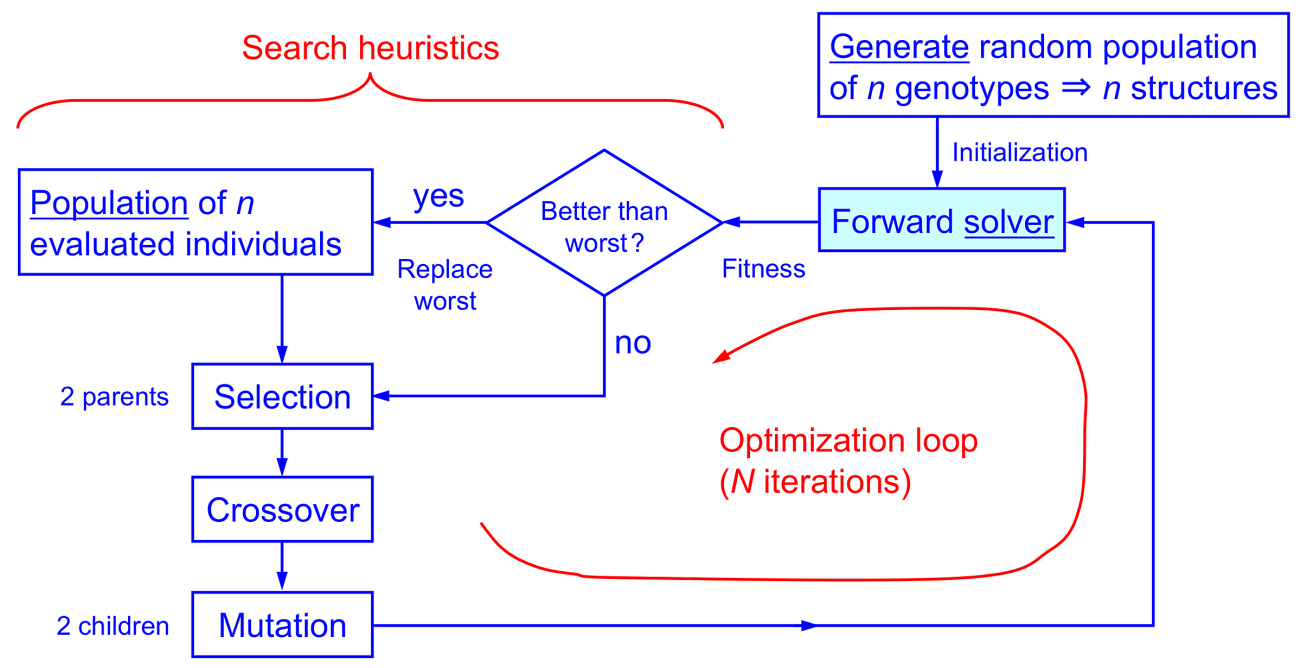

Figure A1. schematic of the breeder evolutionary algorithm's working principle.

\section{References}

1. Chopra, K.L.; Paulson, P.D.; Dutta, V. Thin-film solar cells: An overview. Prog. Photovolt. Res. Appl. 2004, 12, 69-92. [CrossRef]

2. Poortmans, J.; Arkhipov, V. Thin Film Solar Cells: Fabrication, Characterization and Applications; John Wiley \& Sons: Hoboken, NJ, USA, 2006.

3. Hegedus, S. Thin film solar modules: The low cost, high throughput and versatile alternative to Si wafers. Prog. Photovolt. Res. Appl. 2006, 14, 393-411. [CrossRef]

4. Callahan, D.M.; Munday, J.N.; Atwater, H.A. Solar cell light trapping beyond the ray optic limit. Nano Lett. 2012, 12, 214-218. [CrossRef]

5. Ferry, V.E.; Polman, A.; Atwater, H.A. Modeling light trapping in nanostructured solar cells. ACS Nano 2011, 5, 10055-10064. [CrossRef]

6. Ferry, V.E.; Verschuuren, M.A.; Li, H.B.; Verhagen, E.; Walters, R.J.; Schropp, R.E.; Atwater, H.A.; Polman, A. Light trapping in ultrathin plasmonic solar cells. Opt. Express 2010, 18, A237-A245. [CrossRef]

7. Atwater, H.A.; Polman, A. Plasmonics for improved photovoltaic devices. Nat. Mater. 2010, 9, 205-213. [CrossRef]

8. Catchpole, K.R.; Polman, A. Plasmonic solar cells. Opt. Express 2008, 16, 21793-21800. [CrossRef]

9. Akimov, Y.A.; Koh, W.S. Design of plasmonic nanoparticles for efficient subwavelength light trapping in thin-film solar cells. Plasmonics 2011, 6, 155-161. [CrossRef]

10. Akimov, Y.A.; Koh, W.S.; Sian, S.; Ren, S. Nanoparticle-enhanced thin film solar cells: Metallic or dielectric nanoparticles? Appl. Phys. Lett. 2011, 96, 073111. [CrossRef]

11. Kim, H.; Kim, D. Effect of surface roughness of top cover layer on the efficiency of dye-sensitized solar cell. Sol. Energy 2012, 86, 2049-2055. [CrossRef]

12. Krč, J.; Zeman, M.; Kluth, O.; Smole, F.; Topič, M. Effect of surface roughness of ZnO: Al films on light scattering in hydrogenated amorphous silicon solar cells. Thin Solid Films 2003, 426, 296-304. [CrossRef]

13. Paetzold, U.W.; Moulin, E.; Michaelis, D.; Böttler, W.; Wächter, C.; Hagemann, V.; Meier, M.; Carius, R.; Rau, U. Plasmonic reflection grating back contacts for microcrystalline silicon solar cells. Appl. Phys. Lett. 2011, 99, 181105. [CrossRef]

14. Madzharov, D.; Dewan, R.; Knipp, D. Influence of front and back grating on light trapping in microcrystalline thin-film silicon solar cells. Opt. Express 2011, 19, A95-A107. [CrossRef] 
15. Jalali, M.; Nadgaran, H.; Erni, D. Photon management in c-Si solar cells via plasmonic nanogratings. In CENIDE NanoBio Workshop 2015; Campus Essen, University of Duisburg-Essen: Essen, Germany, 2015.

16. Jalali, M.; Nadgaran, H.; Erni, D. Semi-periodic gratings for broadband absorption in thin film solar cells. In Proceedings of the European Conference on Lasers and Electro-Optics-European Quantum Electronics Conference, Munich, Germany, 21-25 June 2015; paper JSI-2.3 SUN.

17. Jalali, M.; Nadgaran, H.; Erni, D. Semiperiodicity versus periodicity for ultra broadband optical absorption in thin-film solar cells. J. Nanophotonics 2016, 10, 036018. [CrossRef]

18. Martins, E.R.; Li, J.; Liu, Y.; Depauw, V.; Chen, Z.; Zhou, J.; Krauss, T.F. Deterministic quasi-random nanostructures for photon control. Nat. Commun. 2013, 4, 2665. [CrossRef]

19. Pala, R.A.; Liu, J.S.; Barnard, E.S.; Askarov, D.; Garnett, E.C.; Fan, S.; Brongersma, M.L. Optimization of non-periodic plasmonic light-trapping layers for thin-film solar cells. Nat. Commun. 2013, 4, 2095. [CrossRef]

20. Spinelli, P.; Ferry, V.E.; Van de Groep, J.; Van Lare, M.; Verschuuren, M.A.; Schropp, R.E.I.; Atwater, H.A.; Polman, A. Plasmonic light trapping in thin-film Si solar cells. J. Opt. 2012, 14, 024002. [CrossRef]

21. Poxson, D.J.; Kuo, M.; Mont, F.W.; Kim, Y.; Yan, X.; Welser, R.E.; Sood, A.K.; Cho, J.; Lin, S.; Schubert, E.F. High-performance antireflection coatings utilizing nanoporous layers. MRS Bull. 2011, 36, 434-438. [CrossRef]

22. Moghal, J.; Kobler, J.; Sauer, J.; Best, J.; Gardener, M.; Watt, A.A.; Wakefield, G. High-performance, single-layer antireflective optical coatings comprising mesoporous silica nanoparticles. ACS Appl. Mater. Interfaces 2012, 4, 854-859. [CrossRef]

23. Yan, X.; Poxson, D.J.; Cho, J.; Welser, R.E.; Sood, A.K.; Kim, J.K.; Schubert, E.F. Enhanced omnidirectional photovoltaic performance of solar cells using multiple-discrete-layer tailored-and low-refractive index anti-reflection coatings. Adv. Funct. Mater. 2013, 23, 583-590. [CrossRef]

24. Makableh, Y.; Vasan, R.; Sarker, J.; Nusir, A.; Seal, S.; Manasreh, M. Enhancement of GaAs solar cell performance by using a ZnO sol-gel anti-reflection coating. Sol. Energy Mater. Sol. Cells 2014, 123, 178-182. [CrossRef]

25. Oh, S.J.; Chhajed, S.; Poxson, D.J.; Cho, J.; Schubert, E.F.; Tark, S.J.; Kim, D.; Kim, J.K. Enhanced broadband and omni-directional performance of polycrystalline Si solar cells by using discrete multilayer antireflection coatings. Opt. Express 2013, 21, A157-A166. [CrossRef]

26. Jalali, M.; Nadgaran, H.; Erni, D. Design of Silicon Nano-Bars Anti-Reflection Coating to Enhance Thin Film Solar Cells Efficiency. Int. J. Opt. Photonics 2017, 11, 79-86. [CrossRef]

27. Dal, N.L. Optics of Aperiodic Structures: Fundamentals and Device Applications; CRC Press: Boca Raton, FL, USA, 2013.

28. Jalali, M.; Nadgaran, H.; Erni, D. Semi-periodic nanostructures: An apt solution to broadband optical applications. In Proceedings of the International Conference “Days on Diffraction 2018", St. Petersburg, Russia, 4-8 June 2018; pp. 161-162.

29. Erni, D. Periodische und Nichtperiodische Wellenleitergitter-und Laserkavitätskonzepte. Ph.D. Thesis, ETH Zürich, Zürich, Switzerland, 1996.

30. Kroon, L.; Lennholm, E.; Riklund, R. Localization-delocalization in aperiodic systems. Phys. Rev. B 2002, 66, 094204. [CrossRef]

31. Berg, I.D. The algebra of semiperiodic sequences. Mich. Math. J. 1963, 10, 237-239. [CrossRef]

32. Mühlenbein, H. Evolutionary algorithms: Theory and applications. In Local Search in Combinatorial Optimization; Wiley: Hoboken, NJ, USA, 1993.

33. Mühlenbein, H.; Schlierkamp-Voosen, D. Predictive models for the breeder genetic algorithm i. continuous parameter optimization. Evol. Comput. 1993, 1, 25-49. [CrossRef]

34. Erni, D.; Wiesmann, D.; Spühler, M.; Hunziker, S.; Moreno, E.; Oswald, B.; Fröhlich, J.; Hafner, C. Application of evolutionary optimization algorithms in computational optics. ACES J. Spec. Issue Genet. Algorithms 2000, $15,43-60$.

(C) 2019 by the authors. Licensee MDPI, Basel, Switzerland. This article is an open access article distributed under the terms and conditions of the Creative Commons Attribution (CC BY) license (http:/ / creativecommons.org/licenses/by/4.0/). 